\title{
A CMOS Chip Set for Accurate Pulsed Time-of- Flight Laser Range Finding
}

\author{
S. Kurtti, J. Nissinen, J.-P. Jansson and J. Kostamovaara, Senior Member, IEEE \\ University of Oulu, Faculty of Information Technology and Electrical Engineering \\ Circuits and Systems Research Unit \\ Oulu, Finland \\ sami.kurtti@oulu.fi, jan.nissinen@oulu.fi, jussi.jansson@oulu.fi, juha.kostamovaara@oulu.fi
}

\begin{abstract}
A laser rangefinder device based on pulsed timeof-flight (TOF) distance measurement techniques was constructed and tested. Key blocks of the system are the integrated receiver channel and the integrated time-to-digital converter (TDC) fabricated in a 0.35 -um CMOS technology. The receiver-TDC chip set is capable of measuring the time position, rise time and pulse width of incoming optical pulses with ps precision in the amplitude range of more than 1: 50000 . The timing detection is based on leading edge detection in the receiver channel, and the amplitude-dependent timing error is compensated for by utilizing the multichannel TDC. A measurement distance of $100 \mathrm{~m}$ is achieved to a target with a reflectance of about $10 \%$ at the signal level of $S N R=6$, with an optical output power and receiver aperture of $12 \mathrm{~W}$ and $18 \mathrm{~mm}$, respectively.
\end{abstract}

Keywords-Laser radar receiver; Pulsed time-of-flight; Laser rangefinder; Time-to-digital converter

\section{INTRODUCTION}

The time-of-flight (TOF) radars are typically based on either continuous wave $(\mathrm{CW})$ phase comparison method or on the measurement of the transit time $(\Delta \mathrm{T})$ of a short laser pulse to an optically visible target and back to the receiver [1]. In a pulsed TOF the distance is computed as $\mathrm{R}=\left(\Delta \mathrm{T}^{*} \mathrm{c}\right) / 2$, where $\mathrm{c}$ is the speed of flight.

Optical distance measurement principles have important advantages over microwave radars, for example. One of them is the good transversal accuracy (perpendicular to optical axis) due to the collimated transmitted beam. Laser radars have found use in several industrial distance measurement applications, for example in proximity sensors and in positioning of tools, and in automotive applications such as velocity control [2] - [4].

The pulsed TOF method is especially interesting in environmental perception systems where high measurement speed $(<\mathrm{ms})$ is needed and the dynamics of the received echo may vary in a very wide range $(>1: 10000)$. This measurement principle enables cm-level precision even with a single transmitted pulse to distances up to tens of meters to noncooperative targets. Moreover, the integrated circuit technology suggested here for laser radar electronics is typically low-cost, and thus this technology is potentially interesting for highvolume applications.

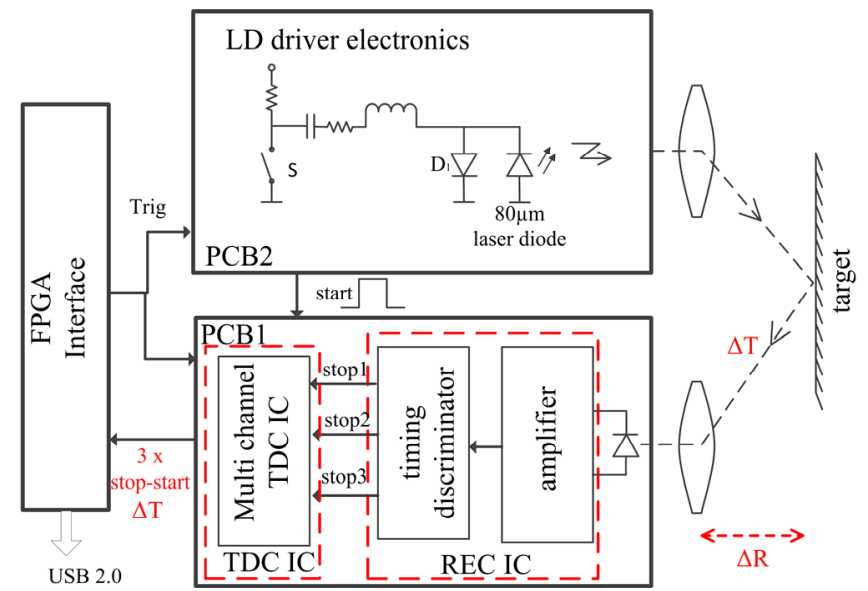

Fig. 1. Pulsed TOF laser rangefinder.

A pulsed TOF laser rangefinder typically consists of a semiconductor laser diode based transmitter, which produces laser pulses having a peak power and pulse width of 10 to $30 \mathrm{~W}$ and 3 to $4 \mathrm{~ns}$. The typical receiver realization consists of an avalanche photo diode (APD) detector, amplifying stages, a timing comparator, and a time-to-digital converter (TDC). A block diagram of the laser rangefinder device used in this work including also the necessary optics and FPGA interface is depicted in Fig. 1.

For practical reasons, the laser pulse width is usually a few nanoseconds, which corresponds to $\sim 0.5 \mathrm{~m}$ in air. Obviously, the detection of a specific timing point from the received laser pulse is needed. The key challenge is to be able to detect the time position of the received ns-scale pulse accurately, with a maximum timing walk of $1 \mathrm{~cm}(\sim 70 \mathrm{ps})$ within the required dynamic range. The dynamic range of the received optical echoes can be larger than 1: 100000 in traffic applications due to the large variation of reflection characteristics of the objects (e.g. shining metal surfaces, which sometimes can be covered by mud).

In this work, we have developed two integrated circuits, an analogue receiver channel and a digital multi-channel TDC, which are realized in a standard $0.35 \mu \mathrm{m}$ CMOS technology and whose performance fulfills the required functionalities of a pulsed time-of-flight laser receiver. The CMOS integrated

The authors would like to acknowledge financial support from Academy of Finland (Center of Excellence in Laser Scanning Research, contract no. 307362, and contracts no. 255359 and 263705). 
receiver channel detects a weak optical echo and generates logic level timing signals for the multi-channel TDC, which measures the time intervals between the transmitted and received pulses as well as the pulse widths and rise times used for the timing walk error compensation [5]. The high level of integration paves the way for low-cost, and miniaturized LIDAR sensors, which are needed in emerging new applications [6].

In Section II, we represent the functionality and the performance of the receiver-TDC chip set. In Section III, the construction of the laser radar system including the optics, the transmitter and the receiver are presented. Finally, some measurement results are given from the measurement track using the developed laser radar.

\section{RECEIVER CHANNEL AND TDC}

\section{A. Receiver channel}

An integrated receiver channel was implemented in a standard $0.35-\mu \mathrm{m}$ CMOS technology [5]. The required bandwidth $\left(\mathrm{BW} \sim 0.35 / \mathrm{t}_{\mathrm{r}}\right.$ ) for the receiver channel was set based on the used laser pulse with a rise time of $\sim 1.5 \mathrm{~ns}$, see Fig. 2.

The receiver channel uses the leading edge timing discrimination to discriminate the timing point from the incoming laser pulse. The timing comparator is triggered only when the received pulse exceeds a predetermined threshold voltage $\left(\mathrm{V}_{\text {th }} \mathrm{L}\right)$ which is related to the noise level of the receiver [7] as shown in Fig. 3. The normal practice is to use a threshold voltage that is $5 \ldots 7$ times the RMS-noise value according to the desired rate of false alarms. Unfortunately, the leading edge timing discrimination principle will produce a relatively large timing walk error (nanosecond range) in its basic configuration for the received optical echo whose amplitude varies a lot [8].

However, by knowing the relation between the walk and measured pulse width/rise time based on the calibration measurement, the timing error can be compensated for from the distance result. The receiver channel gives the required timing information in the form of three stop signals (stop1, stop2, stop3) from the received pulse for the multi-channel TDC, as can be seen in Fig. 3. The time domain walk compensation is explained in more detail in [5]. The exact value of the upper threshold voltage $\left(\mathrm{V}_{\text {th }} \mathrm{U}\right)$ in Fig. 3 is not critical from the timing walk compensation point of view. It is only important to use the same threshold voltages for the calibration measurement (measurement of compensation tables) and for the actual distance measurement in which the compensation table is used.

The receiver channel consists of a low noise preamplifier, post amplifiers, 2 parallel timing comparators and bias circuitry, as is shown in the simplified block in Fig. 4. The threshold voltages $\left(\mathrm{V}_{\text {th } \mathrm{L}}\right.$ and $\left.\mathrm{V}_{\text {th } \mathrm{U}}\right)$ of the timing comparators in the receiver channel are controlled via on-chip 6-bit digitalto-analog converters (DACs). The timing comparators operate in the non-clocked configuration. The receiver channel is programmed by means of a SPI bus (FPGA interface) which controls the on-chip Serial-In Parallel-Out (SIPO) register.

The measured characteristics of the receiver channel are: a signal bandwidth of $\sim 250 \mathrm{MHz}$, a transimpedance of $\sim 100 \mathrm{k} \Omega$, the input referred rms noise current of the receiver $\sim 100 \mathrm{nA}$ (Cin,total $\sim 3 \mathrm{pF}$ ) and the power consumption of about $180 \mathrm{~mW}$. The use of walk compensation enabled a timing accuracy of $\pm 4 \mathrm{~mm}$ over a dynamic range $\sim 1: 100000$. The size of the receiver chip is $4 \mathrm{~mm}^{2}$ and it is enclosed in a QFN36 package.

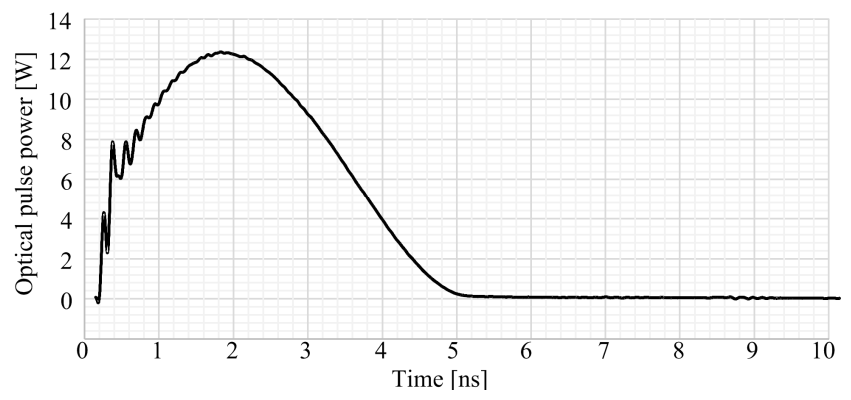

Fig. 2. Optical pulse shape (905D1S2J03Y with MOS pulse driver) of the transmitter recorded with a $25 \mathrm{GHz}$ optical measuremnt head.
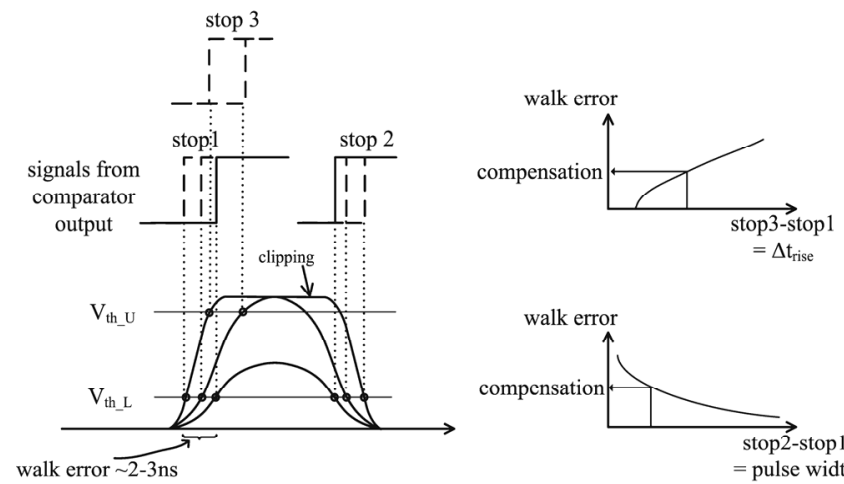

Fig. 3. Principle of timing walk compensation based on the measurements of pulse width and rise time.

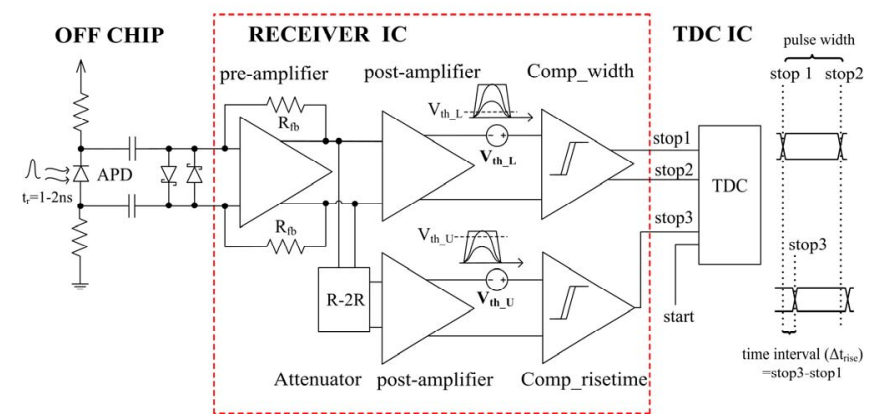

Fig. 4. Simplified block diagram of the receiver IC.

\section{B. Time-to-digital converter}

The multi-channel TDC circuit (10 parallel channels), composed of the blocks depicted in Fig. 5, was fabricated in standard $0.35-\mu \mathrm{m}$ CMOS process. TDC solves time intervals accurately between the start and 3 timing signals (stops) from 
the receiver IC. Start signal for the TDC is composed from the driving current of the laser diode in transmitter as shown in Fig. 1. TDC measures 3 time intervals $\Delta \mathrm{t}_{1}$ (start-stop1), $\Delta \mathrm{t}_{2}$ (start-stop2) and $\Delta \mathrm{t}_{3}$ (start-stop3)). $\Delta \mathrm{t}_{1}$ defines an actual distance information (including walk error), $\Delta \mathrm{t}_{2}-\Delta \mathrm{t}_{1}$ corresponds to the pulse width and $\Delta \mathrm{t}_{3}-\Delta \mathrm{t}_{1}$ to the rise time information, used for the timing walk error compensation.

The multi-channel TDC (10 parallel channels) is based on a counter and delay line interpolation in two nested, different resolution levels. The measurement core is presented in Fig. 6.

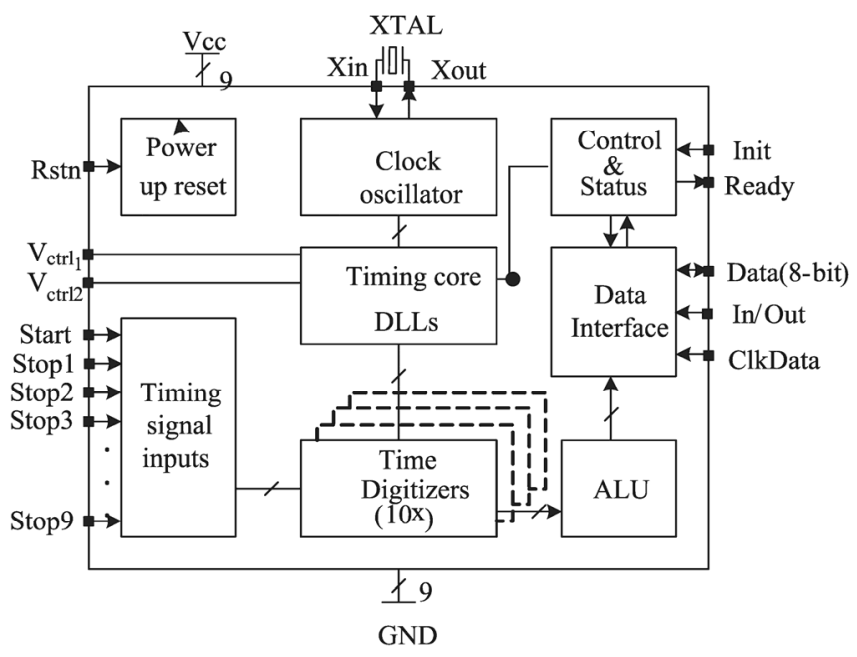

Fig. 5. The block diagram of the TDC.

In the TDC, a $20 \mathrm{MHz}$ input reference signal is multiplied internally up to $240 \mathrm{MHz}$ with a multiplying delay-locked loop (MDLL) [9] which acts as the first 'coarse' interpolation level with 4-bit resolution. The MDLL consists of a delay adjustable delay line, phase detector and charge pump. The phase detector monitors and the charge pump adjusts the delay line delay so that the cycle time of the delay line (multiplied by 12) corresponds the cycle time of the reference crystal. The delay locking stabilizes the measurement against process, voltage and temperature variations (PVT). The arriving start signals sets on the 7-bit counter and counts the rounds of the MDLL between the start and stop signals, providing a $530 \mathrm{~ns} \quad(\sim 80 \mathrm{~m})$ measurement range with $4.2 \mathrm{~ns}$ resolution.

The MDLL delay line interpolates the locations of timing signals (start and 9 stops) within the counter cycle time. The delay line with identical successive delay elements generates 16 even size phases with 260 ps resolution, when the multiplied reference signal propagates in the MDLL with 240 $\mathrm{MHz}$ frequency. The timing signals store the prevailing state of the delay line into a register bank, from where the first interpolation level results can be coded.

The second 'fine' interpolation level continues the interpolation and solves the locations of the timing signals within the first interpolation level delay element delay thus the time interval between the asynchronous stop and the synchronized edge at the first interpolation level is solved. One delay is divided into 32 LSB phases with parallel load capacitor-scaled delay elements [9]. The small time differences ( $8.1 \mathrm{ps}$ ) between the parallel elements are achieved by a small difference in their capacitive loads.

The multi-channel TDC was fabricated in a standard 0.35$\mu \mathrm{m}$ CMOS process and enclosed in a plastic QFN48 package. As the receiver channel generates 3 stop signals 3 of 9 TDCs stop channels are used with a 10 ps resolution. 8-bit bidirectional data input/output lines are controlled via the FPGA interface. The power consumption of the TDC is $\sim 150 \mathrm{~mW}$ and the power supply voltage $3.3 \mathrm{~V}$.

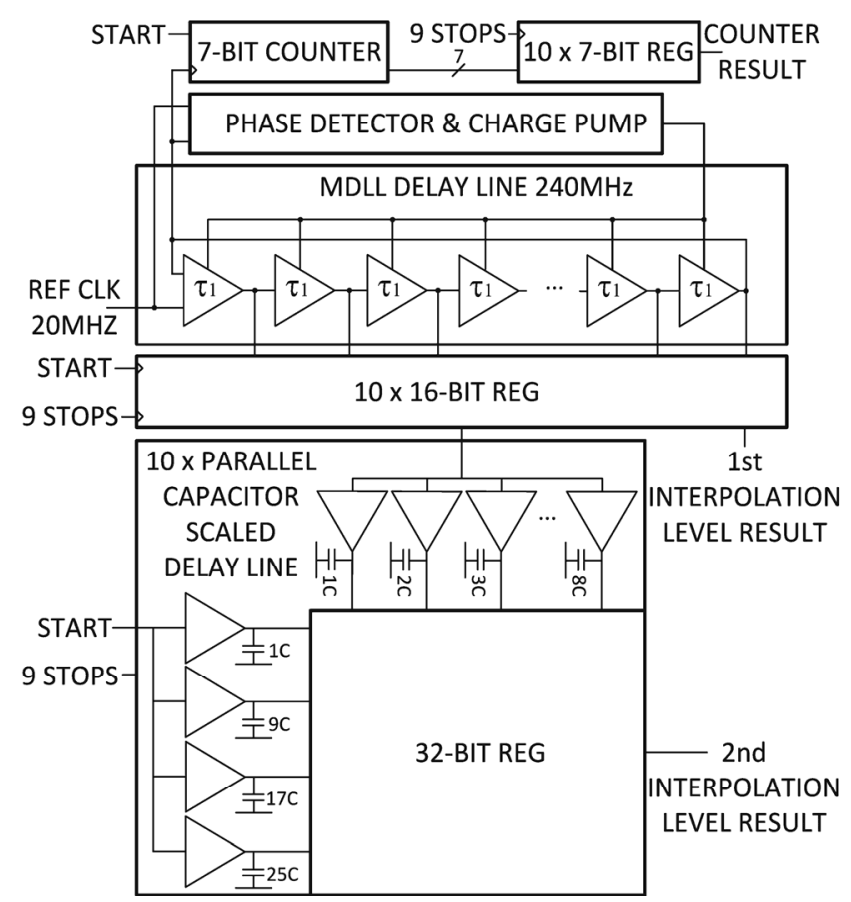

Fig. 6. TDC measurement core based on counter and 2-level interpolation.

\section{CONSTRUCTION AND MEASUREMENTS}

The simplified measurement setup is shown in Fig. 1. The receiver channel, multi-channel TDC and laser transmitter were controlled using an Opal Kelly XEM6001 FPGA board.

The transmitter consists of a laser diode (905D1S2J03Y from Laser Components) and a laser diode driver, which was realized with a MOS switch and an LRC transient-based pulse shape control [10]. The laser diode produces an optical output signal with a measured peak output power of $18 \mathrm{~W}(12 \mathrm{~W}$ after optics), at a wavelength of $905 \mathrm{~nm}$ and with a half-value width of $\sim 3 \mathrm{~ns}$, as shown in Fig. 2. The pulsing rate of the transmitter can be set up to $\sim 100 \mathrm{kHz}$. In addition, the transmitter PCB consists of an ECL comparator (ADCMP553) which is used to generate a start signal for the TDC from the driving current of the laser diode.

Fig. 7 shows the photograph of the receiver PCB including the avalanche photodiode (APD) and both the receiver and TDC circuits. The receiver unit was integrated to a laser rangefinder device which utilizes parallel optics, as shown in 
photograph in Fig. 8. On the receiver side an avalanche photo diode (AD230-8 TO52S1 from the First Sensor) with an active area of $230 \mu \mathrm{m}$ was used. The focal lengths of the transmitter and receiver optics are $30 \mathrm{~mm}$ and $20 \mathrm{~mm}$, respectively. As a result, the divergence of the laser beam is equal to $\sim 3 \mathrm{mrad}$ $(80 \mu \mathrm{m} / 30 \mathrm{~mm})$ giving a spot size of $9 \mathrm{~cm}$ at 30 meters. Correspondingly, the field of view of the receiver is $11.5 \mathrm{mrad}$ $(230 \mu \mathrm{m} / 20 \mathrm{~mm})$.

The lower threshold voltage $\left(\mathrm{V}_{\text {th } ~}\right)$ and the upper threshold voltage $\left(\mathrm{V}_{\text {th }} \mathrm{U}\right)$ were set to $\sim 70 \mathrm{mV}(\mathrm{SNR} \sim 7)$ and $\sim 500 \mathrm{mV}$, respectively in the calibration and in the laser radar measurements.

Laser radar measurements were carried out on a measurement track with a maximum distance of $34 \mathrm{~m}$, as depicted in Fig. 9. The wide dynamic range for the reflected echo was achieved by using different kind of materials as the target. The single-shot distribution $(5,000$ shots $)$ of the black cardboard (reflection coefficient $(\rho) \sim 0.12$ ) at a distance of $34 \mathrm{~m}$ was $74 \mathrm{ps}$ (11 mm in distance) at the signal level of SNR $\sim 50$, as shown in Fig. 10 (solid line). This includes the jitter of the TDC ( $\sim 10 \mathrm{ps})$, jitter induced by the compensation and the jitter of the receiver channel (due to noise). The jitter of the start signal is low since it is generated from the driving current of the laser diode in transmitter side.

For a comparison, the single-shot distribution of walkcompensated results from a highly directional diamond grade reflector $(\rho>>1)$ at distance of $34 \mathrm{~m}$ is also shown as dashed line in Fig. 10. This signal level corresponds to SNR level of $>$ 10000 resulting single shot precision of $\sim 15 \mathrm{ps}(2 \mathrm{~mm}$ in distance) which is already near to the jitter of the TDC.

Using the known parameters above, a maximum distance of $\sim 100 \mathrm{~m}$ can be predicted for the SNR level of $\sim 6$ in laser range finding.

\section{CONCLUSIONS}

In conclusion, a CMOS chip-set was developed, fabricated and tested for a pulsed TOF laser radar. The chip set includes two high-performance integrated circuits which realize the receiver channel and the time interval measurement functionalities, respectively. It should be noted that in principle, the receiver and TDC could also be integrated on the same IC chip based on the $0.35-\mu \mathrm{m}$ CMOS technology. In addition, the power consumption could be scaled down by using the shut-down principle while the laser transmitter is not operating.

The developed chip set can detect optical echoes with a typical length of 3-4 ns in a dynamic range of more than 1:100 000 with an accuracy better than $100 \mathrm{ps}$, and can serve numerous emerging applications in the field of TOF laser radars. Moreover, by measuring to the highly reflective surfaces a single shot precision of $\sim 10$ ps can be achieved with a chip-set realized in a non-aggressive CMOS technology.

The receiver-TDC chip-set achieves better performance compared to recently published papers [11], [12]. The integrated receiver for pulsed LIDAR system reported in [11] utilizes gain control structures to widen the dynamic range.
However, the resulting dynamic range (1:1600) in [11] is narrower than in the proposed receiver. In [12] the dynamic range of 1: 12000 has been achieved but the measurement accuracy is limited to few tens of centimeters only whereas the accuracy achieved in this work was $1 \mathrm{~cm}$, even in a wider dynamic range. It should be noted that the designed and measured receiver-TDC chip-set includes also the high performance time interval measurement unit which is in any case needed and is not shown in [11], [12]. The integrated receiver channel, multi-channel TDC and the time-domain walk compensation presented here, paves the way to miniaturized laser radar sensor systems.

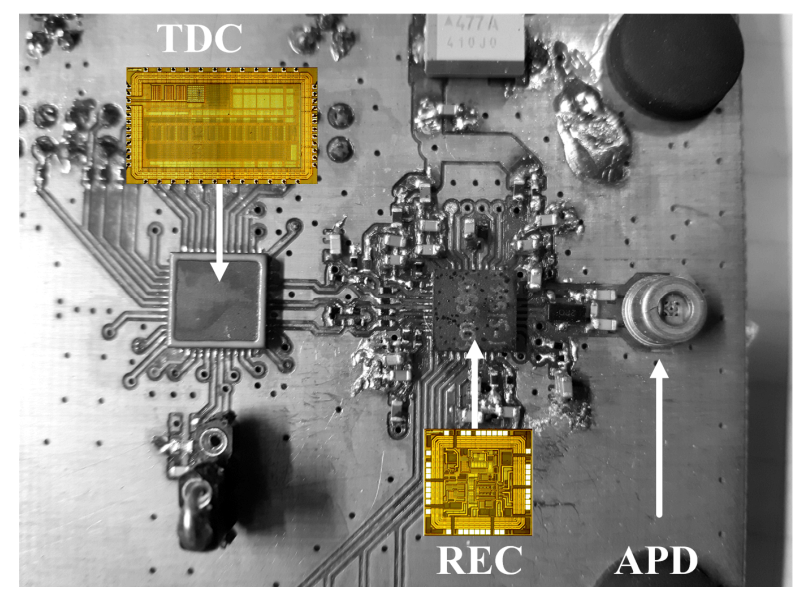

Fig. 7. Photograph of the measurement PCB including the receiver and TDC chips.

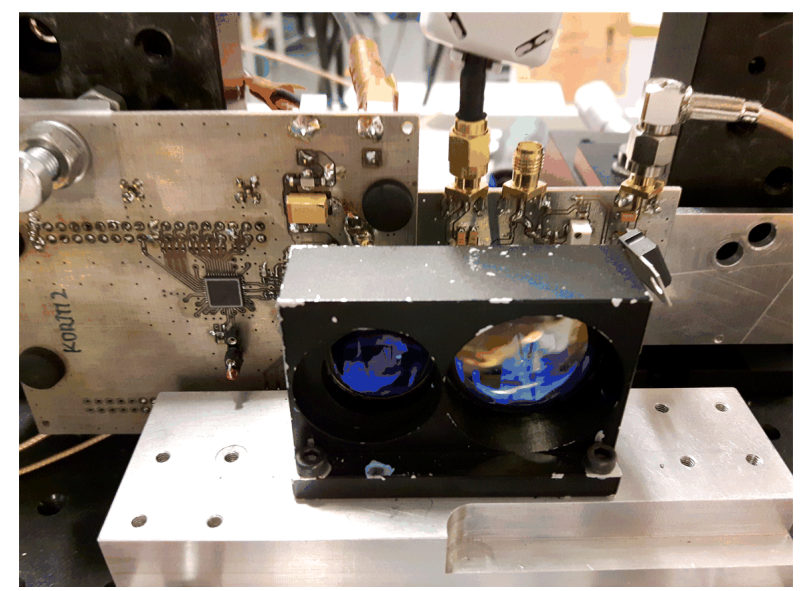

Fig. 8. Transmitter and receiver optics. 


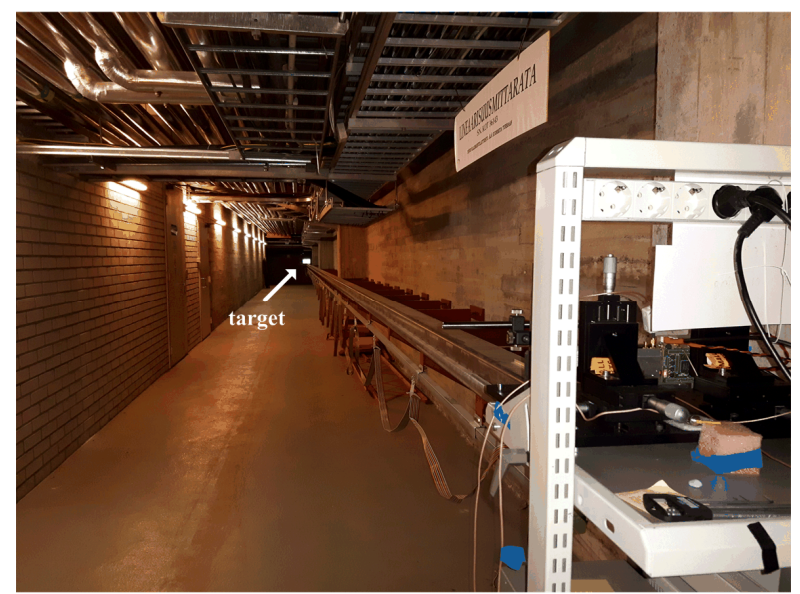

Fig. 9. Pulsed TOF laser radar measurement track.

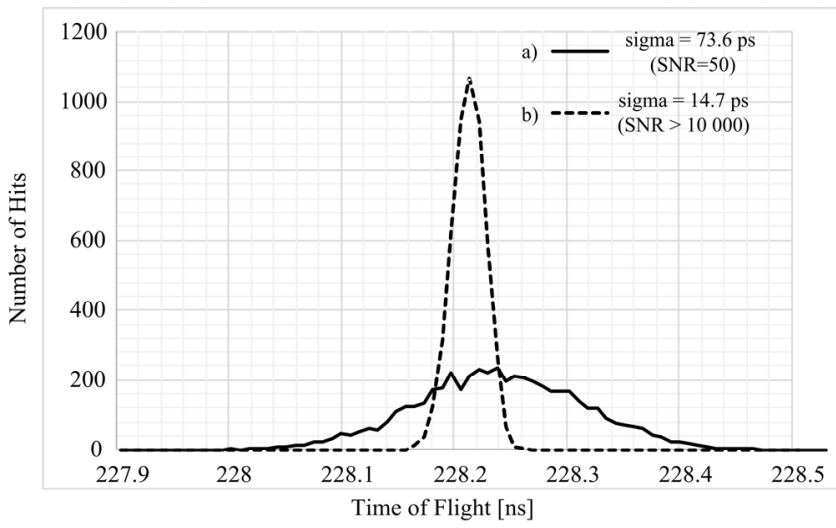

Fig. 10. Single-shot distribution of 5,000 hits of walk-compensated results at $34 \mathrm{~m}$ measured for a) a black piece of cardboard $(\rho \sim 0.12)$ and b) a directional diamond grade reflective sheet $(\rho>>1)$.

\section{REFERENCES}

[1] S. Donati, "Electro-Optical Instrumentation: Sensiong and Measuring With Lasers," Upper Saddle River, NJ, USA: Prentice-Hall, 2004.

[2] M. Xuesong, D. Inoue, S. Kato, and M. Kagami, “Amplitude-modulated laser radar for range and speed measurement in car application," IEEE Trans. Intell. Transp. Syst., vol. 13, no.1, pp. 408-413, Mar. 2012.

[3] D. C. Carmer and L. M. Peterson, "Laser radar in robotics," Proc. IEEE, vol. 84, no. 2, pp. 299-320, Feb. 1996.

[4] S. Velupillai and Guvench L., Laser scanners for driver-assitance systems in intelligent vehicles," IEEE Cont. Syst. Mag., vol. 29, no. 2, 17-19, Apr. 2009.

[5] S. Kurtti, J. Nissinen, and J. Kostamovaara, ”A wide dynamic range CMOS laser radar receiver with a time-domain walk error compensation scheme", IEEE Trans. Circuits Syst. I, Reg. Papers, vol. 64, no. 3, pp. 550-561, Mar. 2017.

[6] V. C. Coffey, "Imaging in 3-D: Killer apps coming soon to a device near you!" Opt. Photon. News, vol 25., no. 6, pp. 36-43, 2014.

[7] H. N. Burns, C. G. Christodoulou, and G. D. Boreman, "System design of a pulsed laser rangefinder," Opt. Eng., vol. 30, no. 3, pp. 323-329, 1991.

[8] S. Kurtti and J. Kostamovaara, "An integrated laser radar receiver channel utilizing a time-domain walk error compensation scheme", IEEE Trans. on Inst. and Meas. (TIM), vol. 60, no. 1, pp. 146-157, Jan 2011.

[9] J. Jansson, A. Mantyniemi, and J. Kostamovaara, " A CMOS time-todigital converter with better than 10 ps single-shot precision," IEEE J. Solid-State Circuits, vol. 41, pp. 1286-1296, Jun. 2006.

[10] L. Hallman, J. Huikari, J. Kostamovaara, "A high-speed/power laser transmitter for single photon imaging applications", SENSORS, 2014 IEEE, pp. 1157-1160, 2-5 Nov. 2014.

[11] T.-H. Ngo, C.-H. Kim, Y. J. Kwon, J. S. Ko, D.-B. Kim, and H.-H Park, "Wideband receiver for three-dimensional ranging LADAR system," IEEE Trans. Circuits Syst. I, Reg. Papers, vol. 60, no. 2, pp. 448-456, Feb. 2013.

[12] H.-S. Cho, C.-H. Kim, and S.-G. Lee, "A high-sensitivity and low-walk error LADAR reiceiver for military application," IEEE Trans. Circuits Syst. I, Reg. Papers, vol. 61, no. 10, pp. 3007-3015, Oct. 2014. 